\section{Avaliação da implantação da iniciativa hospital amigo da criança no Rio de Janeiro, Brasil}

\section{An evaluation of the implantation of the baby-friendly hospital initiative in Rio de Janeiro, Brazil}

Maria Inês Couto de Oliveira 1

Zulmira Maria de Araújo Hartz 2

Vivianne Cavalcanti do Nascimento 3

Kátia Silveira da Silva 4

\section{Abstract}

Objectives: to evaluate the implantation of the baby-friendly hospital initiative in Rio de Janeiro.

Methods: an evaluative study was carried out in 2009 at seven baby-friendly hospitals (BFHs) and eight non-accredited hospitals (NAHs) forming part of the Brazilian National Health System. Evaluation of the structure (Steps 1 and 2) was carried out by way of observation and interviews with 215 staff members. Evaluation of the process (steps 3 to 10) involved interviews with a representative sample of 461 pregnant women, 687 mothers on the wards and 148 mothers with a newborn in a neonatal unit. The degree of implantation was assessed in terms of the fulfillment of each step and the parameters covered by it. The correlation between the degree of implantation and outcomes was analyzed using linear regression: breastfeeding $(B F)$ in the first hour of life, exclusive breastfeeding (EBF) and the satisfaction of the women with the care provided.

Results: the degree of implantation varied from 9 to 5 steps ( $90.6 \%$ to $70.1 \%$ of parameters) completed at the BFHs and 5 to 1 steps (76.1\% to $43.9 \%$ of parameters) at the NAHs. A significant linear correlation was found between the degree of implantation, expressed in terms of steps and parameters, respectively and $B F$ in the first hour of life ( $r=0.78$ and $r=0.74), E B F(r=0.72$ and $r=0.69)$, and satisfaction $(r=0.69$ and $r=0.73)$.

Conclusions: both forms of evaluation were shown to be consistent with the results. The BFHs performed better than the NAHs and there is therefore a need for investment in the sustainability of this initiative.

Key words Breast feeding, Hospital, Program evaluation, Quality of health care, Newborn, Unified Health System.
1 Departamento de Epidemiologia e Bioestatística. Instituto de Saúde da Comunidade. Universidade Federal Fluminense. Rua Marquês do Paraná, 303. $3^{\circ}$ andar. Centro. Niterói, RJ, Brasil.

E-mail: marinesco@superig.com.br

2 Instituto de Higiene e Medicina Tropical, Universidade Nova de Lisboa. Lisboa, Portugal.

3 Programa de Pós-Graduação em Saúde Coletiva. Instituto de Saúde da Comunidade. Universidade Federal Fluminense. Niterói, RJ, Brasil. 4 Pós-graduação em Saúde da Criança e da Mulher. Unidade de Pesquisa Clínica. Instituto Fernandes Figueira/Fiocruz. Rio de Janeiro, RJ, Brasil.

\section{Resumo}

Objetivos: avaliar a implantação da Iniciativa Hospital Amigo da Criança no Rio de Janeiro.

Métodos: pesquisa avaliativa realizada em 2009, com sete hospitais credenciados (HAC) e oito não (HNC), do SUS. Avaliação de estrutura (Passos 1 e 2) realizada por observação e entrevista a 215 profissionais. Avaliações de processo (Passos 3 a 10) e resultado compreenderam entrevista em amostra representativa de 461 gestantes, 687 mães em alojamento conjunto e 148 mães com recém-nascido em unidade neonatal. $O$ grau de implantação foi avaliado segundo o cumprimento de cada passo e de parâmetros compreendidos nestes passos. A correlação entre grau de implantação e desfechos foi analisada por meio de regressão linear: aleitamento materno (AM) na primeira hora, AM exclusivo (AME) e satisfação das mulheres com o apoio recebido.

Resultados: os graus de implantação variaram de 9 a 5 passos (90,6\% a 70,1\% dos parâmetros) cumpridos nos HAC e de 5 a 1 passo (76,1\% a 43,9\% dos parâmetros) nos HNC. Foi encontrada correlação linear significativa entre o grau de implantação, expresso em passos e parâmetros, respectivamente, $e$ $o$ AM na primeira hora $(r=0,78$ e $r=0,74)$, o $A M E$ $(r=0,72$ e $r=0,69)$, e a satisfação $(r=0,69$ e $r=0,73)$.

Conclusões: ambas as formas de avaliação mostraram-se consistentes com os resultados. Os HAC apresentaram um desempenho superior aos $H N C$, sendo necessário um investimento na sustentabilidade desta iniciativa.

Palavras-chave Aleitamento materno, Hospital, Avaliação de programas e projetos de saúde, Qualidade da assistência à saúde, Recém-nascido, Sistema Único de Saúde 


\section{Introdução}

As evidências sobre os benefícios do aleitamento materno (AM), em especial quando exclusivo no primeiro semestre de vida, vêm se acumulando nas últimas décadas. ${ }^{1}$ Até o final da década de 1970 o desmame precoce vinha sendo praticado em larga escala, contribuindo para a desnutrição e morbimortalidade infantis. ${ }^{2}$ Para a reversão deste quadro, a World Health Organization (WHO) e o Fundo das Nações Unidas para a Infância (UNICEF) em 1979 promoveram uma reunião conjunta sobre alimentação infantil que gerou o "Código Internacional de Comercialização de Substitutos do Leite Materno". Também no Brasil surgem ações pró-amamentação, tendo a "Política Nacional de Incentivo ao Aleitamento Materno" sido lançada em 1981.3

As políticas voltadas à assistência hospitalar se iniciaram em 1986, quando a Assembléia Mundial da Saúde condenou as doações de substitutos do leite materno a maternidades. Em 1989 a WHO/UNICEF divulgaram a "Declaração Conjunta sobre o Papel dos Serviços de Saúde e Maternidades", criando em seguida a Iniciativa Hospital Amigo da Criança (IHAC), mobilizando os serviços obstétricos para a adoção dos "Dez Passos para o Sucesso do Aleitamento Materno".3 Após adequação das normas e auto-avaliação pelos hospitais, avaliadores externos realizam pré-avaliação e avaliação global do cumprimento dos passos, culminando no credenciamento desses serviços quando todos os dez passos são considerados cumpridos. Para que os passos sejam considerados cumpridos pela IHAC, é estipulado que pelo menos $80 \%$ da clientela recebam a assistência preconizada, sendo que para alguns parâmetros compreendidos nos passos este critério varia de 50 a $80 \% .4$

A “Cúpula em Favor da Infância” em 1990 estabeleceu como meta para a década credenciar 50\% dos hospitais com mais de 1000 partos/ano como "Hospital Amigo da Criança" (HAC). ${ }^{3}$ Apesar desta meta ainda não ter sido alcançada, temos mais de 20.000 HAC no mundo, distribuídos em 156 países, sendo 335 no Brasil, onde ocorrem 21\% dos nascimentos do país. No Estado do Rio de Janeiro há dezesseis HAC, oito situados na capital, sete deles com demanda superior a 1000 partos/ano. O título de HAC e sua manutenção têm se revelado conquistas árduas, e o monitoramento deste processo é um desafio global. Desde o ano 2000 o Ministério da Saúde, juntamente às Secretarias de Saúde, vêm buscando reavaliar a cada três anos os hospitais credenciados. ${ }^{5}$ O Brasil estipula o cumprimento de critérios complementares, relativos à assistência prestada ao binômio mãe-filho, também a serem monitorados. Nas reavaliações tri anuais, quando o HAC não cumpre integralmente os "Dez Passos" e os critérios complementares, são dados três meses de prazo, renováveis, para serem feitas adequações, apenas ocorrendo descredenciamento se as exigências não forem cumpridas após três reavaliações. No final da década passada foi iniciado um sistema informatizado de auto-monitoramento anual pelos próprios $\mathrm{HAC}$, proposto pela WHO/UNICEF e endossado pelo Ministério da Saúde, ainda não avaliado. 6

A evidência da efetividade da IHAC em estender a duração da amamentação exclusiva já está estabelecida. ${ }^{7-9}$ No entanto, o Brasil ainda é um dos países da América Latina com menor prevalência de aleitamento materno exclusivo (AME), 10 apesar da contribuição das políticas governamentais 3 para a tendência ascendente observada. A mediana do AME no Brasil era de um mês em 1996, passando a 2,2 meses em 2006.11 A mesma tendência foi observada no município do Rio de Janeiro onde, no mesmo período, a prevalência de AME em menores de seis meses aumentou de 13,8\% para 33,3\%.12 Porém, ainda estamos distantes dos seis meses de AME preconizados pela WHO.13 Outro indicador, a proporção de crianças amamentadas na primeira hora de vida, também evoluiu, passando de $32,8 \%$ em 1996 para 43,0\% em 2006.11

Avaliar a implantação da IHAC permite identificar procedimentos já estabelecidos e obstáculos existentes, aspectos relevantes para o planejamento e aprimoramento de suas ações. Este estudo teve como objetivo avaliar a implantação da IHAC no município do Rio de Janeiro, considerando a influência do grau de implantação na prática do AM e na satisfação da clientela.

\section{Métodos}

Trata-se de pesquisa avaliativa de análise de implantação do Tipo 2, que verifica a influência da variação na implantação da IHAC sobre os efeitos observados. ${ }^{14}$ Os dois primeiros passos desta iniciativa correspondem à estrutura do hospital para a prática dos passos seguintes, que remetem ao processo de promoção, proteção e apoio ao AM. Os resultados aferidos foram o AM ao nascimento, a prevalência de AME (ou uso de suplementos por motivos médicos $)^{4,15}$ até a alta hospitalar e a satisfação da clientela com o apoio recebido para amamentar. 16

Foi construído um modelo teórico de avaliação (Figura 1) que elucida as situações problema que conduziram à criação da IHAC, as atividades 
conduzidas nos hospitais sobre as populações alvo, os desfechos de curto, médio e longo prazo, e as variáveis intervenientes relacionadas ao contexto organizacional: a complexidade do hospital, a presença de comitê de AM e o tempo decorrido entre a última avaliação externa e a coleta de dados.

A pesquisa teve como cenário os quinze hospitais com mais de 1000 partos/ano vinculados ao Sistema Único de Saúde (SUS) no município do Rio de Janeiro, que concentram cerca de $94 \%$ dos partos realizados pelo SUS, 17 sendo sete $\mathrm{HAC}$ e oito não credenciados (HNC).

O tamanho da amostra foi determinado de forma estratificada, sendo os estratos os hospitais. Como não havia estudo semelhante atual para basearmos o cálculo, optou-se por considerar uma prevalência arbitrária de $50 \%$ de cumprimento de cada passo. Prevalência de $50 \%$ foi utilizada também como fator de ponderação para cada estrato, pois garante o maior tamanho de amostra possível para nível de erro e confiança controlados. 18

Para um nível de erro de $5 \%$ e de confiança de $95 \%$, obteve-se um tamanho mínimo de 458 gestantes, distribuídas segundo a quantidade de consultas pré-natais realizadas por cada hospital no segundo semestre de 2008. Foram transcritos dados dos prontuários, sendo incluídas aquelas com 28 ou mais semanas de gestação e com duas ou mais consultas de pré-natal naquele hospital. Cerca de $10 \%$ das entrevistadas foram gestantes internadas há mais de 48 horas por alguma intercorrência.

Para um nível de erro de $5 \%$ e de confiança de $99 \%$, obteve-se um tamanho mínimo de 687 mães em alojamento conjunto, distribuídas entre os estratos segundo a quantidade de partos em 2008. Foram incluídas as mães com filhos nascidos vivos há mais de 24 horas, e excluídas aquelas com contraindicação à amamentação, como sorologia positiva para o vírus da imunodeficiência humana (HIV). 19 As entrevistadas foram selecionadas por sorteio sistemático a partir da ficha do posto de enfermagem. Quando o sorteio selecionava uma mãe com bebê em unidade neonatal há mais de 48 horas, esta era entrevistada, gerando uma amostra de 148 mães de bebês internados. Foi calculada também amostra de 220 profissionais da assistência pré-natal, ao parto e ao recém-nato, proporcional ao quantitativo de cada hospital.

Foram utilizados questionários de reavaliação externa da IHAC15 para a avaliação do grau de implantação da IHAC por hospital. A avaliação dos passos 1 e 2 foi conduzida por meio de observação dos serviços e de entrevista a profissionais de saúde, o passo 3 por entrevista a gestantes e os passos 4 a
10 por entrevista a mães. O grau de implantação foi pontuado de duas formas: segundo o cumprimento de cada passo e segundo a realização dos parâmetros compreendidos em cada passo. A opção por elencar parâmetros compreendidos em cada passo foi no sentido de expor práticas levadas em consideração para a verificação do cumprimento destes passos. Assim, parâmetros satisfatórios não deixariam de ser computados em detrimento de outros não praticados a contento, e aspectos necessitando de melhoria seriam discriminados daqueles já estabelecidos nos hospitais.

Para o primeiro tipo de pontuação foi calculado o número de passos cumpridos por cada hospital, segundo os critérios estabelecidos pela IHAC, 15 onde são utilizados indicadores agregados ou combinados, e foi computada a proporção de cumprimento de cada passo nos HAC e HNC. Para o segundo tipo de pontuação também foi calculada a proporção de profissionais, gestantes e mães cobertas pelas práticas, porém estes dados foram apresentados de forma desagregada. Os escores do grau de implantação da IHAC por hospital e por categoria hospitalar corresponderam à média da soma dos escores obtidos em cada parâmetro.

Aos questionários de reavaliação da $\mathrm{IHAC} 15$ foram acrescidas perguntas sobre características dos profissionais e sociodemográficas das mulheres, para verificar se o perfil dos entrevistados variou entre HAC e HNC. Foram acrescentadas também questões para aferir a ocorrência de $\mathrm{AM}$ na primeira hora de vida entre mães em alojamento conjunto, a prevalência de AME durante a permanência hospitalar no conjunto das mães e a satisfação das gestantes e mães com o apoio recebido do hospital para amamentar. 16

A coleta de dados foi realizada em cada hospital por profissional de saúde capacitada na IHAC, supervisionada por enfermeira credenciada pelo Ministério da Saúde enquanto avaliadora da IHAC. 4 A coordenadora e as três supervisoras conduziram capacitação teórico-prático de 40 horas para as sete entrevistadoras, e toda a equipe da pesquisa participou de estudo piloto, realizado em julho de 2009, em dois hospitais: um HAC e um HNC. O trabalho de campo ocorreu de agosto a dezembro de 2009.

Foi desenvolvida uma análise bivariada para verificar se o perfil dos entrevistados e os indicadores de resultado apresentaram diferença estatisticamente significativa entre estes dois blocos de hospitais. A regressão linear foi utilizada para analisar a correlação entre o grau de implantação da IHAC, expresso em passos cumpridos e em escores, 


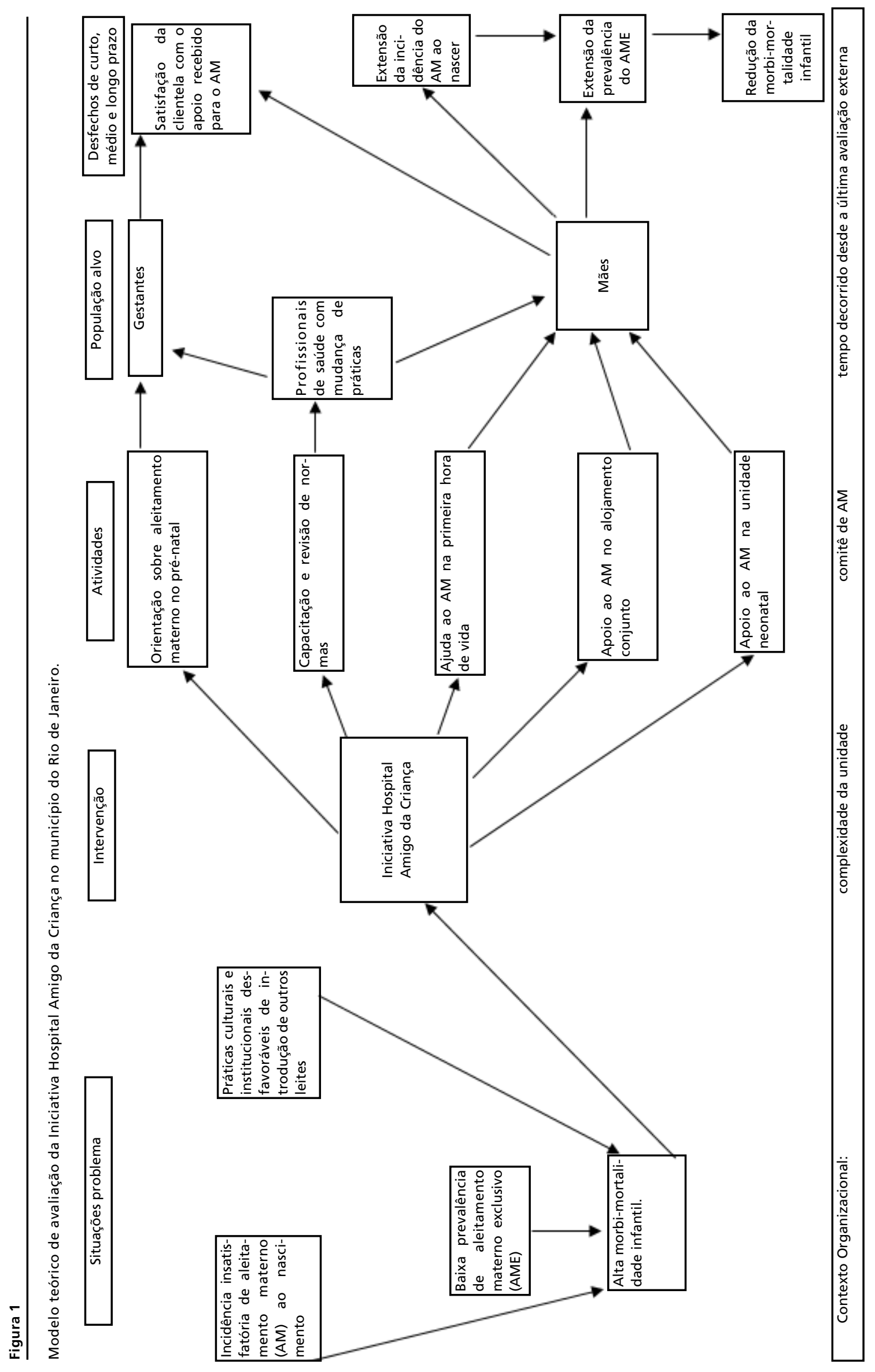


e os indicadores de resultado por hospital, bem como com o tempo decorrido desde a última avaliação externa, sendo utilizado o coeficiente de correlação de Pearson, com nível de significância de 5\%.

Esta pesquisa foi aprovada pelo Comitê de Ética em Pesquisa da Secretaria Municipal de Saúde, parecer $\mathrm{n}^{\circ} 77 \mathrm{~A} / 2009$, sendo os dados colhidos mediante assinatura de Termo de Compromisso Livre e Esclarecido. O Epi-Info foi utilizado para montagem do banco de dados e a análise foi realizada pelo SPSS. 18

\section{Resultados}

Foram entrevistadas as chefias de cada maternidade e 215 profissionais de saúde, 104 em HAC e $111 \mathrm{em}$ HNC. Das 461 gestantes entrevistadas, $272(59,9 \%)$ eram assistidas por HAC e 189 por HNC. Foram entrevistadas 687 mães em alojamento conjunto e 148 com bebês em unidade neonatal, cerca da metade em HAC. O perfil dos entrevistados em HAC e HNC foi semelhante, exceto por haver uma maior proporção de gestantes adolescentes nos HNC (Tabela 1).

A maior parte dos hospitais eram públicos, da rede municipal (7), estadual (4) ou federal (3), com exceção de uma maternidade filantrópica. Destes quinze hospitais, cinco HAC e seis HNC faziam parte do sistema de gestação de alto risco, sendo hospitais de referência materna e/ou fetal. Dois HAC e um HNC eram vinculados a instituições de ensino. Os hospitais apresentaram taxas de cesárea que variaram de $14 \%$ a $53 \%$.

Doze hospitais dispunham de Comitê de AM, responsável pela realização dos cursos de capacitação e pelas solicitações de pré-avaliação ou avaliação global.4,15 O tempo entre a última avaliação externa e a pesquisa variou de três meses a 11 anos e 10 meses, havendo dois hospitais nunca avaliados pela IHAC. O número de passos cumpridos por hospital variou de 9 a 1, com amplitude de 9 a 5 entre HAC e de 5 a 1 entre HNC (Tabela 2). Os HAC apresentaram escores com amplitude de 90,6 a 70,1, com escore médio de 80,4, e os HNC de 76,1 a 43,9, com escore médio de 58,2 (Tabela 3).

$\mathrm{Na}$ avaliação de estrutura, observou-se que todos os HAC e três HNC dispunham de normas adequadas, porém estas não estavam expostas em um dos HAC. Mais de $80 \%$ dos profissionais de HAC receberam treinamento e possuíam conhecimento adequado (Tabela 3), no entanto dois HAC não cumpriam o passo 2, e apenas um HNC o cumpria (Tabela 2).

$\mathrm{Na}$ avaliação de processo, verificou-se que nos
HAC mais de $70 \%$ das gestantes foram informadas sobre o AM (Tabela 3), mas dois HAC não cumpriam o passo 3, enquanto apenas um HNC o cumpria (Tabela 2). O passo 4, relativo ao AM ao nascimento, foi cumprido por apenas quatro HAC (Tabela 2), apesar do contato pele-a-pele ter sido bastante praticado (Tabela 3). A ajuda ao AM no alojamento conjunto e à manutenção da lactação na unidade neonatal alcançou mais de $70 \%$ das mães, porém a orientação sobre frequência da ordenha na unidade neonatal só atingiu a metade das mães (Tabela 3), dois HAC e a maioria dos HNC não cumprindo o passo 5 (Tabela 2). A oferta de suplementos sem indicação clínica atingiu $16,9 \%$ dos recém-natos em alojamento conjunto nos $\mathrm{HAC}$ e $57,4 \%$ nos HNC (Tabela 3 ), cinco HAC e somente um HNC cumprindo o passo 6 (Tabela 2).

Mais de $90 \%$ da clientela recebeu a assistência preconizada pelos passos 7 (praticar o alojamento conjunto) e 9 (não usar bicos) (Tabela 3 ) e apenas um hospital não credenciado, que variou a cada passo, não os cumpriram (Tabela 2). Também o passo 8 (livre demanda) foi incentivado junto a mais de $90 \%$ das mães em alojamento conjunto em HAC e mais de $80 \%$ daquelas em HNC (Tabela 3), porém quatro destes hospitais não o cumpriam (Tabela 2). Já o passo 10 , relativo ao encaminhamento a apoio ao AM pós-alta, foi o menos praticado, pois nenhum hospital o cumpria (Tabela 2). Menos de um quarto das mães assistidas em HNC e menos da metade daquelas em HAC receberam esta informação, apesar de três HAC terem alcançado uma cobertura superior a $70 \%$ (Tabela 3 ).

A avaliação de resultado mostrou que cerca da metade das mães em alojamento conjunto havia amamentado na primeira hora após o parto, sendo que nos HAC a cobertura foi o dobro da encontrada nos HNC. A proporção de recém-natos que recebeu AME durante a permanência hospitalar (ou fez uso de suplemento por motivos médicos $)^{4,15}$ foi de $64,2 \%$, maior nos HAC $(81,6 \%)$ em relação aos HNC (46,2\%). Expressaram satisfação com o apoio recebido do hospital para amamentar $76,3 \%$ das mulheres (Tabela 3). Foi observada uma correlação linear positiva significativa $(p<0,05)$, tanto entre o número de passos cumpridos, quanto entre o escore, e todos os indicadores de resultado por hospital: amamentação na primeira hora de vida, aleitamento materno exclusivo durante a permanência hospitalar, e satisfação das mulheres com o apoio recebido para amamentar. O tempo decorrido desde a última avaliação externa em cada hospital mostrou uma correlação negativa robusta, tanto com o número de passos cumpridos, quanto com o escore (Figura 2). 
Tabela 1

Características dos entrevistados em Hospitais Amigos da Criança e Hospitais Não Credenciados do Sistema Único de Saúde no município do Rio de Janeiro, 2009.

\begin{tabular}{|c|c|c|c|c|c|}
\hline \multirow{2}{*}{ Características } & \multicolumn{2}{|c|}{ HAC } & \multicolumn{2}{|c|}{ HNC } & \multirow{2}{*}{$p$} \\
\hline & $\mathrm{n}$ & $\%$ & $\mathrm{n}$ & $\%$ & \\
\hline \multicolumn{6}{|c|}{ Profissionais de saúde $(n=215)$} \\
\hline \multicolumn{6}{|c|}{ Profissão } \\
\hline Médico & 29 & 27,9 & 33 & 29,7 & \multirow{2}{*}{0,765} \\
\hline Não médico & 75 & 72,1 & 78 & 70,3 & \\
\hline \multicolumn{6}{|c|}{ Tempo de trabalho (anos) } \\
\hline Até 5 & 50 & 48,1 & 41 & 36,9 & \multirow{2}{*}{0,099} \\
\hline 6 ou mais & 54 & 51,9 & 70 & 63,1 & \\
\hline \multicolumn{6}{|l|}{ Gestantes $(n=461)$} \\
\hline \multicolumn{6}{|l|}{ Idade } \\
\hline Adolescente & 49 & 18,0 & 52 & 27,5 & \multirow{2}{*}{0,015} \\
\hline Adulta & 223 & 82,0 & 137 & 72,5 & \\
\hline \multicolumn{6}{|l|}{ Cor } \\
\hline Branca & 86 & 31,6 & 52 & 27,5 & \multirow{2}{*}{0,344} \\
\hline Não branca & 186 & 68,4 & 137 & 72,5 & \\
\hline \multicolumn{6}{|l|}{ Escolaridade (anos) } \\
\hline Até 7 & 73 & 26,8 & 54 & 28,6 & \multirow{2}{*}{0,682} \\
\hline 8 ou mais & 199 & 73,2 & 135 & 71,4 & \\
\hline \multicolumn{6}{|l|}{ Trabalho } \\
\hline Não & 103 & 37,9 & 81 & 42,9 & \multirow{2}{*}{0,282} \\
\hline $\operatorname{sim}$ & 169 & 62,1 & 108 & 57,1 & \\
\hline \multicolumn{6}{|l|}{ Paridade } \\
\hline Primigesta & 129 & 47,4 & 87 & 46,0 & \multirow{2}{*}{0,768} \\
\hline 1 ou mais filhos & 143 & 52,6 & 102 & 54,0 & \\
\hline \multicolumn{6}{|c|}{ Mães em alojamento conjunto $(n=687)$} \\
\hline \multicolumn{6}{|c|}{ Idade } \\
\hline Adolescente & 45 & 12,9 & 47 & 13,9 & \multirow{2}{*}{0,697} \\
\hline Adulta & 304 & 87,1 & 291 & 86,1 & \\
\hline \multicolumn{6}{|l|}{ Cor } \\
\hline Branca & 83 & 23,8 & 88 & 26,0 & 0,495 \\
\hline Não branca & 266 & 76,2 & 250 & 74,0 & 0,495 \\
\hline Escolaridade (anos) & & & & & \\
\hline Até 7 & 107 & 30,7 & 113 & 33,4 & 0,436 \\
\hline 8 ou mais & 242 & 69,3 & 225 & 66,6 & 0,436 \\
\hline Trabalho & & & & & \\
\hline Não & 170 & 48,7 & 158 & 46,7 & 0,234 \\
\hline $\operatorname{sim}$ & 179 & 51,3 & 180 & 53,3 & \\
\hline Paridade & & & & & \\
\hline Primípara & 172 & 49,3 & 156 & 46,2 & 0.412 \\
\hline 2 ou mais filhos & 177 & 50,7 & 182 & 53,8 & \\
\hline Mães com bebê em ur & & & & & \\
\hline Idade & & & & & \\
\hline Adolescente & 16 & 21,3 & 22 & 30,1 & 0,220 \\
\hline Adulta & 59 & 78,7 & 51 & 69,9 & \\
\hline
\end{tabular}


Características dos entrevistados em Hospitais Amigos da Criança e Hospitais Não Credenciados do Sistema Único de Saúde no município do Rio de Janeiro, 2009.

\begin{tabular}{|c|c|c|c|c|c|}
\hline \multirow{2}{*}{ Características } & \multicolumn{2}{|c|}{ HAC } & \multicolumn{2}{|c|}{ HNC } & \multirow{2}{*}{$p$} \\
\hline & $\mathrm{n}$ & $\%$ & $\mathrm{n}$ & $\%$ & \\
\hline \multicolumn{6}{|l|}{ Cor } \\
\hline Branca & 25 & 33,3 & 23 & 31,5 & \multirow{2}{*}{0,812} \\
\hline Não branca & 50 & 66,7 & 50 & 68,5 & \\
\hline \multicolumn{6}{|l|}{ Escolaridade (anos) } \\
\hline Até 7 & 27 & 36,0 & 33 & 45,2 & \multirow{2}{*}{0,254} \\
\hline 8 ou mais & 48 & 64,0 & 40 & 54,8 & \\
\hline \multicolumn{6}{|l|}{ Trabalho } \\
\hline Não & 30 & 40,0 & 33 & 45,2 & \multirow{2}{*}{0,522} \\
\hline Sim & 45 & 60,0 & 40 & 54,8 & \\
\hline \multicolumn{6}{|l|}{ Paridade } \\
\hline Primípara & 31 & 41,3 & 40 & 54,8 & \multirow{2}{*}{0,101} \\
\hline 2 ou mais filhos & 44 & 58,7 & 33 & 45,2 & \\
\hline
\end{tabular}

$\mathrm{HAC}=$ Hospitais Amigos da Criança; HNC= Hospitais Não Credenciados. 


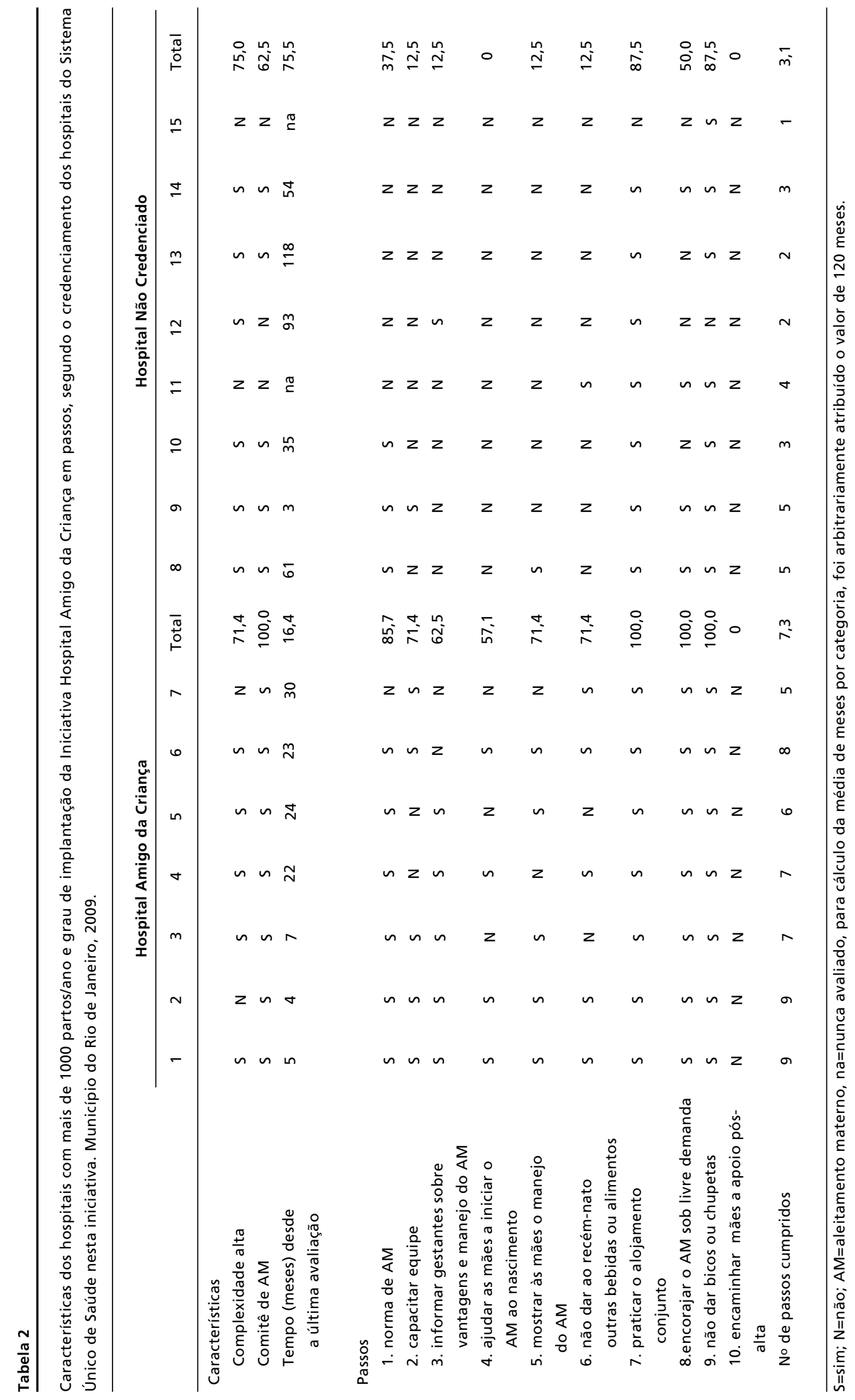




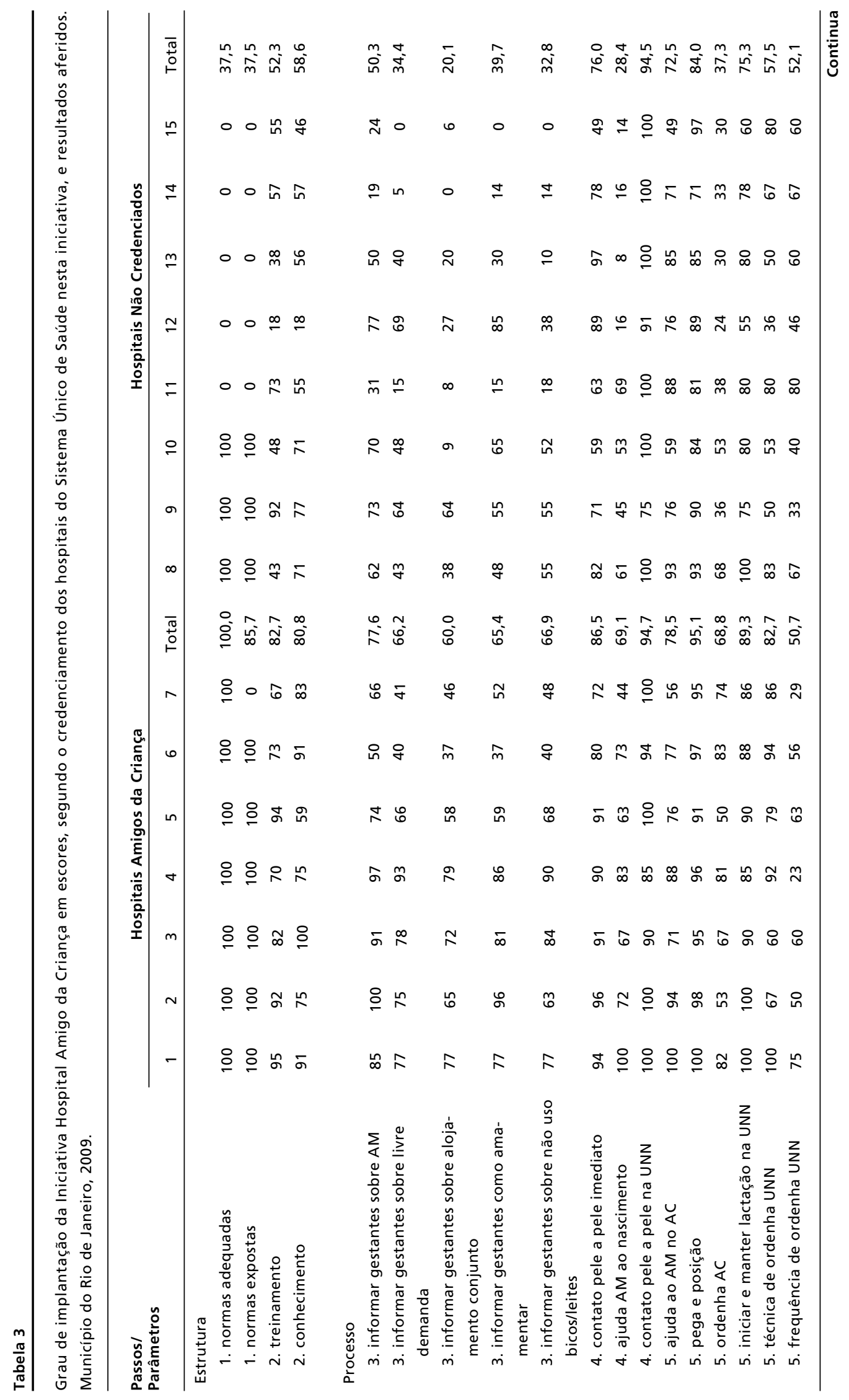




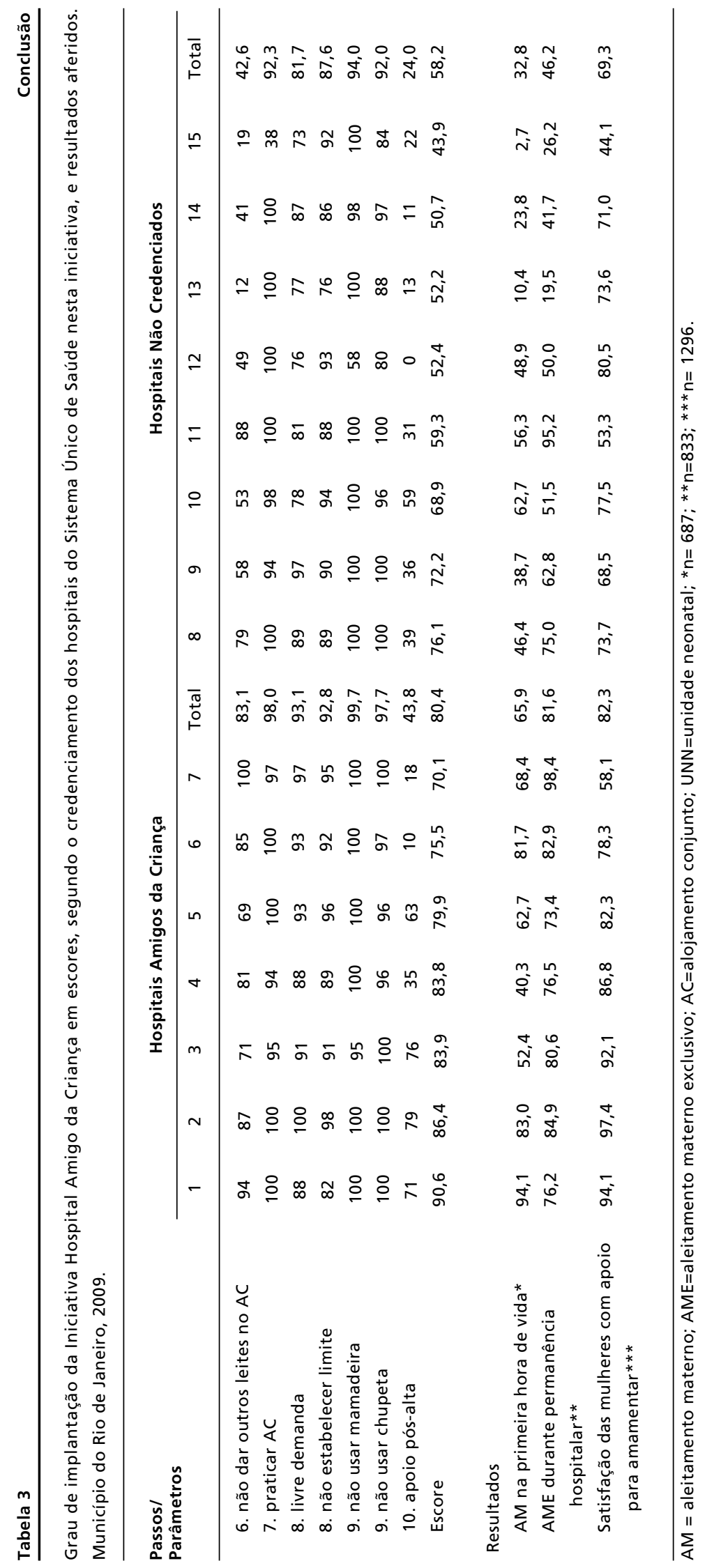


Correlação entre o grau de implantação da Iniciativa Hospital Amigo da Criança por hospital, expresso em passos cumpridos e em escores, e o tempo desde a última avaliação externa, o aleitamento materno (AM) na $1^{\text {a }}$ hora, o AM exclusivo e a satisfação com o apoio para amamentar. Município do Rio de Janeiro, 2009.
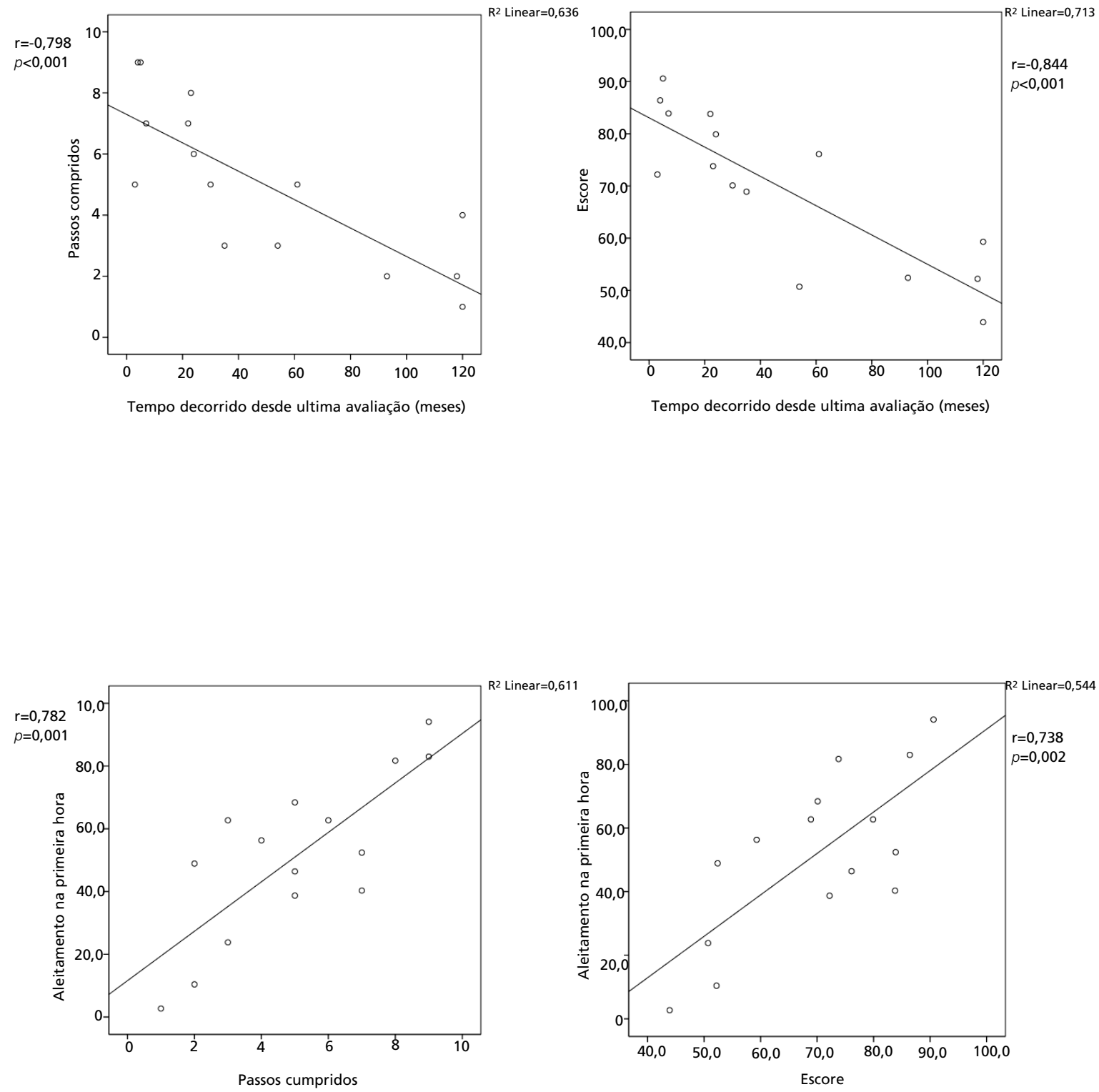
Correlação entre o grau de implantação da Iniciativa Hospital Amigo da Criança por hospital, expresso em passos cumpridos e em escores, e o tempo desde a última avaliação externa, o aleitamento materno (AM) na $1^{\text {a }}$ hora, o AM exclusivo e a satisfação com o apoio para amamentar. Município do Rio de Janeiro, 2009.
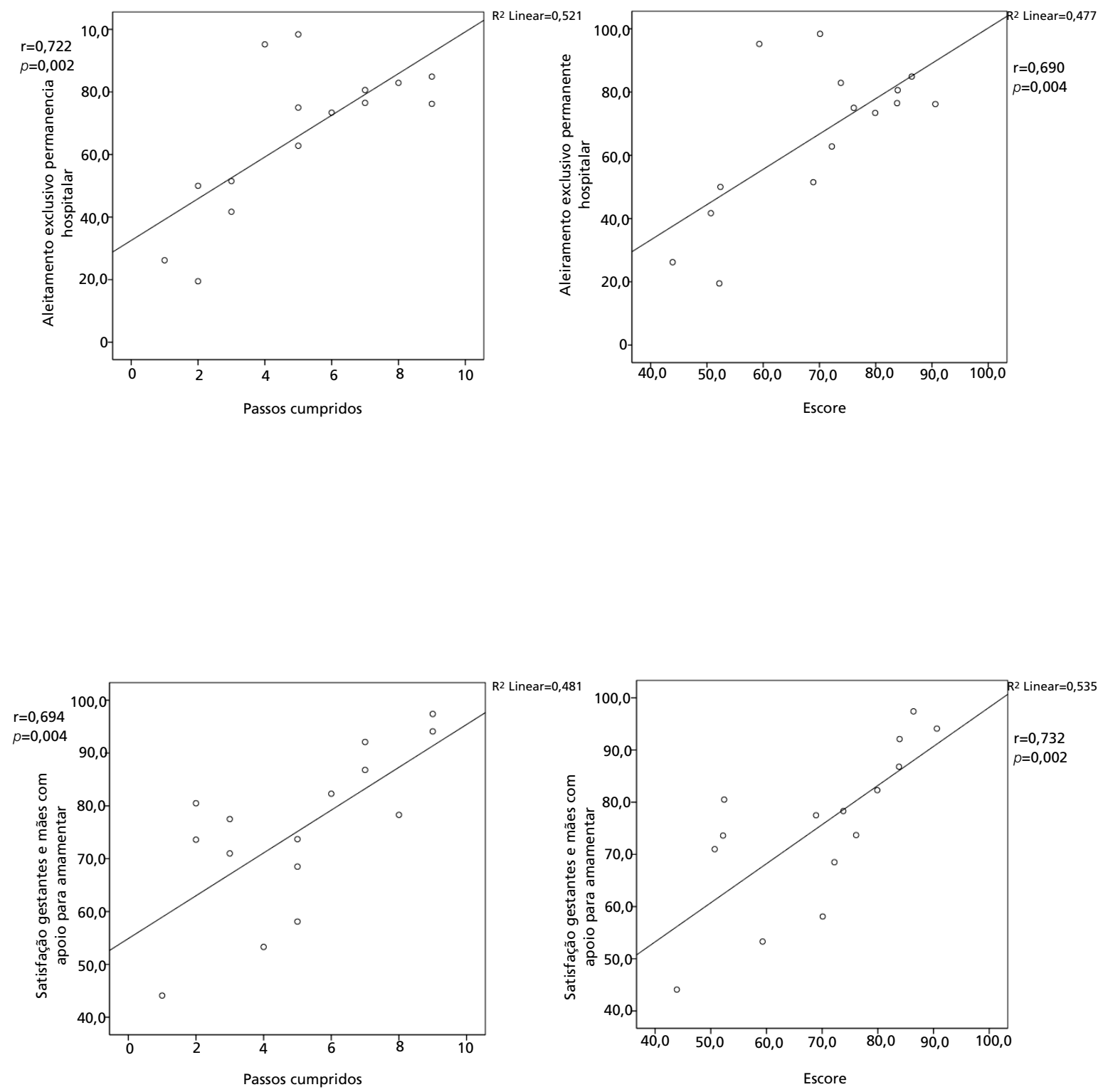

* O tempo decorrido entre a última avaliação e a pesquisa nos dois hospitais que nunca passaram por uma avaliação externa foi arbitrariamente atribuído em 120 meses. 


\section{Discussão}

Todos os hospitais com mais de 1000 partos/ano no município do Rio de Janeiro estavam envolvidos com a IHAC, contemplando vários parâmetros e cumprindo pelo menos um passo, porém ainda havia uma maternidade onde o alojamento conjunto não era praticado desde o nascimento, direito assegurado no Brasil por lei.20

Esta avaliação se deu num cenário de pesquisa, diferentemente das reavaliações externas, onde os hospitais são avisados com antecedência, e buscam aprimorar suas práticas. Esta pode ser uma explicação para o fato de nenhum HAC estar cumprindo integralmente os "Dez Passos para o Sucesso do Aleitamento Materno" na ocasião do trabalho de campo. Porém, a grande quantidade de parâmetros praticados, e o cumprimento por todos os HAC de pelo menos a metade dos passos, evidenciaram que o processo de conquista deste indicador de qualidade é tão transformador que mesmo no cenário cotidiano dos serviços a maior parte destas práticas se mantém. No entanto, a importância do monitoramento das mesmas ficou demonstrada, pois o grau de implantação variou fortemente de maneira inversa com o tempo decorrido desde a última avaliação externa. Foi interessante também verificar que alguns HNC apresentaram um grau de implantação semelhante ao de hospitais já credenciados, o que parece indicar que há hospitais em processo avançado de conquista do título, que apresentam práticas equivalentes àqueles cuja avaliação externa ocorreu menos recentemente, onde possivelmente ocorreu um retrocesso na implantação de algumas ações.

Neste estudo foram avaliados três indicadores de resultado, a partir dos dados colhidos nas entrevistas, diferentemente da IHAC, que utiliza apenas o AME durante a permanência hospitalar aferido por dados pregressos. ${ }^{4}$ Este indicador é influenciado pela complexidade do hospital, que não apresentou uma distribuição homogênea no cenário desta pesquisa, apesar de sua distribuição equivalente entre os dois blocos de hospitais. É um indicador sujeito a viés de informação pela dificuldade em discriminar se o uso de suplemento teve indicação clínica, porém mostrou uma correlação linear moderada positiva com o grau de implantação da IHAC, corroborando sua validade. Uma associação de magnitude e direção semelhantes foi encontrada entre o grau de implantação e a satisfação da clientela, um indicador de fácil aferição e já validado. ${ }^{21} \mathrm{O}$ desfecho $\mathrm{AM}$ na primeira hora de vida, também suscetível a viés de informação, pois se baseia na estimativa materna do tempo decorrido entre o parto e a primeira mamada, mostrou da mesma forma uma correlação positiva moderada com o grau de implantação da IHAC.

Ambas as formas de pontuação dos hospitais mostraram que o grau de implantação se associou significativamente aos desfechos aferidos, denotando consistência. No entanto, vale realçar as suas diferenças. Na pontuação por parâmetros desagregados, ficou mais fácil perceber que aspectos necessitavam ser melhorados e aqueles já difundidos nos hospitais, parâmetros satisfatórios não deixaram de ser contabilizados em detrimento de outros não praticados a contento, e foi possível distinguir os passos quase cumpridos daqueles deficitários. Os passos avaliados através de vários parâmetros ganharam mais peso, apesar da sua importância não poder ser aferida apenas pela quantidade de parâmetros contemplados na avaliação. Na pontuação que sinalizou o cumprimento dos passos, os parâmetros não foram discriminados, mas a visualização dos passos cumpridos ficou mais evidente, sendo atribuído o mesmo peso a todos os passos.

Em reavaliação nacional do cumprimento dos Dez Passos da IHAC, realizada em 2002, foi verificado, de forma consistente ao presente estudo, que os passos 7 (praticar o alojamento conjunto) e 9 (não dar bicos) foram os de maior adesão. $\mathrm{Na}$ época, o passo 3 (informar gestantes sobre AM) foi o menos cumprido, seguido pelo 2 (capacitação da equipe) e pelos passos 10 (encaminhamento a apoio pós-alta), 4 (ajuda à amamentação ao nascimento) e 5 (mostrar às mães o manejo do AM).22

A importância da orientação ao AM no pré-natal e do apoio pós-alta já foram documentados, ${ }^{21}$ no entanto, é difícil de ser percebida pelos profissionais que atuam nos hospitais. As equipes que prestam assistência pré-natal e ao parto são distintas, após a alta hospitalar estes profissionais não têm mais contato com as mães, e não se percebe uma preocupação com uma linha de cuidado integrada que considere todo o ciclo gravídico-puerperal. ${ }^{23}$ Talvez isto seja uma fonte de explicação para o baixo desempenho da maior parte dos hospitais nos passos 3 e 10, pois os profissionais não vivenciam o impacto de suas práticas. Em maternidades de Palmares, ${ }^{24}$ e Salvador 25 foram avaliados os passos 4 a 10 , e o passo 10 também foi o menos praticado.

Outra explicação para o baixo desempenho do passo 10 é o viés de informação. Apesar de terem sido entrevistadas mães cujo parto ocorrera há mais de 24 horas, como este estudo apresentou um desenho transversal, não foi possível saber se o encaminhamento a apoio pós-alta teria sido prestado após a entrevista. Em avaliações externas a aferição deste procedimento pode ser realizada até no 
primeiro dia pós-parto, pois é preconizado que este encaminhamento seja disponibilizado à mãe durante a internação, e não no momento da alta, quando sua atenção pode estar voltada para outras questões, prejudicando a assimilação da informação. ${ }^{4}$

O presente estudo não comportou a análise dos determinantes da deficiência encontrada na prática de passos ou parâmetros individuais. Porém, vale referir que o passo 4 foi pouco praticado, possivelmente em função de fatores como a ausência do resultado oportuno do teste rápido anti-HIV, que vem postergando a primeira mamada, 26 e aumentando o uso de suplementos, relativo ao passo 6.27 Apesar do uso não indicado de suplementos em recém-natos nos hospitais credenciados ter sido inferior a $25 \%$, como preconizado pela IHAC, 4 ainda foi superior ao encontrado em HAC de outros cenários, como a Rússia (11,1\%).28

Cabe ainda mencionar outro limite desta investigação. O cumprimento dos passos foi aferido com base nos parâmetros elencados na Tabela 3, que correspondem à maioria, mas não à totalidade dos procedimentos verificados durante uma reavaliação externa formal. 15

Um aspecto a ser ressaltado é que a amostra de cada hospital foi representativa da clientela assistida, e a amostra total foi representativa do conjunto de hospitais com mais de 1000 partos/ano do município do Rio de Janeiro, refletindo, portanto, o panorama da assistência prestada pelo SUS à quase totalidade das gestantes de risco, parturientes e puérperas neste município. A utilização de diversos desfechos permitiu avaliar a consistência entre o grau de implantação, pontuado de duas formas, e o resultado desta intervenção, não só em relação à prática do
AM ao nascimento e do AME, indicadores já amplamente utilizados em pesquisas nacionais, ${ }^{11}$ como também à satisfação da mulher com o apoio recebido para amamentar. Foi assim incorporada a ótica da mulher ao processo de avaliação, que não deve ser vista apenas como cliente destes serviços, mas sim como a atora principal do processo de parturição e amamentação.

Nesta pesquisa foi observado que já existe uma prática sistematizada de avaliação dos $\mathrm{HAC}$, pois todos haviam sido reavaliados num período inferior a três anos, revelando a institucionalização do processo de avaliação da IHAC. No entanto, valeria a pena avaliar também a proposta da WHO/UNICEF de auto-monitoramento anual, que permite um papel ativo das equipes hospitalares na manutenção das práticas inerentes à IHAC. 6 Cabe ressaltar a importância que tem desempenhado o apoio governamental22,29 no processo de implantação da IHAC, sendo necessário, no entanto, um investimento na sustentabilidade desta iniciativa para garantir o acesso universal das mães às práticas de promoção, proteção e apoio ao AM preconizadas.

\section{Agradecimentos}

Os autores gostariam de agradecer ao $\mathrm{CNPq}$ pelo apoio financeiro fornecido através do Edital $\mathrm{n}^{\circ}$ $57 / 2008$ à pesquisa que deu origem a este artigo. Gostariam de agradecer também aos membros da equipe de pesquisa Gisele Peixoto Barbosa, Tânia Maria Brasil Esteves, Abilene do Nascimento Gouvêa e Fátima Maria Trigo da Paz pelo apoio no trabalho de campo e na devolução dos resultados da pesquisa aos hospitais avaliados.

\section{Referências}

1. Toma TS, Rea MF. Benefícios da amamentação para a saúde da mulher e da criança: um ensaio sobre as evidências. Cad Saúde Pública. 2008; 24 (Supl.2): S235-46.

2. Victora CG, Smith PG, Vaughan JP, Nobre LC, Lombardi C, Teixeira AMB, Fuchs SMC, Moreira LB, Gigante LP Barros FC. Evidence for protection by breast-feeding against infant deaths from infectious diseases in Brazil. Lancet. 1987; 2: 319-22.

3. Rea MF. Reflexões sobre a amamentação no Brasil: de como passamos a 10 meses de duração. Cad Saúde Pública. 2003; 19 (Supl.1): S37-45.

4. WHO/UNICEF (World Health Organization, The United Nations Children's Fund). The global criteria for the WHO/UNICEF Baby-Friendly Hospital Initiative. Geneva; 1992.

5. Brasil. Ministério da Saúde. Iniciativa Hospital Amigo da Criança. Área Técnica de Saúde da Criança e Aleitamento Materno. Departamento de Ações Programáticas
Estratégicas/ Secretaria de Atenção à Saúde. [on line] Brasília, DF; 2010 [acesso em 27 out 2011]. Disponível em: http://portal.saude.gov.br/portal/arquivos/pdf/ relatorioihacatualizado.pdf

6. Toma TS, Rea MF. La Iniciativa Hospital Amigo de la Niñez y la herramienta computarizada para su monitoreo y sustentabilidad. Rev Panam Salud Publica. 2011; 30: 505-6.

7. Braun MLG, Giugliani ERJ, Soares MEM, Giugliani C, Oliveira AP, Danelon CMM. Evaluation of the impact of the baby-friendly hospital initiative on rates of breastfeeding. Am J Public Health. 2003; 93: 1277-9.

8. Kramer MS, Chalmers B, Hodnett ED, Sevkovskaya Z, Dzikovich I, Shapiro S, Collet JP, Vanilovich I, Mezen I, Ducruet T, Shishko G, Zubovich V, Mknuik D, Gluchanina E, Dombrovskiy V, Ustinovitch A, Kot T, Bogdanovich N, Ovchinikova L, Helsing E. Promotion of Breast feeding Intervention Trial (PROBIT): a randomized trial in the Republic of Belarus. JAMA. 2001; 285: 413-20. 
9. Lutter CK, Pérez-Escamilla R, Segall A, Sanghvi T, Teruya $\mathrm{K}$, Wickham C. The effectiveness of a hospital-based program to promote exclusive breast-feeding among lowincome women in Brazil. Am J Public Health. 1997; 87: 659-63.

10. Pérez-Escamilla R. Breast feeding patterns in nine Latin America and Caribbean countries. Bull Pan Am Health Organ. 1993; 27: 32-42.

11. Brasil. Ministério da Saúde. Pesquisa Nacional de Demografia e Saúde da Criança e da Mulher - PNDS 2006: dimensões do processo reprodutivo e da saúde da criança / Ministério da Saúde, Centro Brasileiro de Análise e Planejamento. Brasília, DF; 2009.

12. Castro IRR, Engstrom EM, Cardoso LO, Damião JJ, Rito RVFV, Gomes MASM. Tendência temporal da amamentação na cidade do Rio de Janeiro: 1996-2006. Rev Saúde Pública. 2009; 43: 1021-9.

13. WHO (World Health Organization). The optimal duration of exclusive breastfeeding: results of a WHO systematic review. Note for the press $\mathrm{N}^{\circ} 7$. Geneva; 2001.

14. Denis JL, Champagne F. Análise de implantação. In: Hartz ZMA, org. Avaliação em saúde: dos modelos conceituais à prática na análise de implantação de programas. Rio de Janeiro: Fiocruz; 1997. p. 49-88.

15. Brasil. Ministério da Saúde. Módulo 5 - Avaliação e reavaliação externas, OMS/UNICEF. Seção 5.3 Orientações e instrumentos de reavaliação externa. Brasília, DF; 2009.

16. Donabedian A. The quality of care. How can it be assessed? JAMA. 1988; 260: 1743-8.

17. Brasil. Site DATASUS. [acesso em 15 set 2011]. Disponível em: www.datasus.gov.br

18. Cochran WG. Sampling Techniques. Wiley Series. In: Probability and Statistics. Ed: IE-WILEY; 1977.

19. Dunn TDT, Newell ML, Ades AE, Peckham CS. Risk of human immunodeficiency virus type 1 transmission through breastfeeding. Lancet. 1992; 340: 585-8.
20. Brasil. Lei n ${ }^{\circ} 8069$ de 13 de julho de 1990. Estatuto da Criança e do Adolescente. Brasília, DF: Diário Oficial da República Federativa do Brasil. Seção I. 16 de julho de 1990. p. 13563-77.

21. Oliveira MIC, Camacho LAB, Souza IEO. Promoção, proteção e apoio à amamentação na atenção primária à saúde no Estado do Rio de Janeiro, Brasil: uma política de saúde pública baseada em evidência. Cad Saúde Pública. 2005; 21: 1901-10.

22. Araújo MFM, Schmitz BAS. Reassessment of BabyFriendly Hospitals in Brazil. J Hum Lact. 2007; 23: 246-52.

23. Malta DC, Merhy EE. O percurso da linha do cuidado sob a perspectiva das doenças crônicas não transmissíveis. Interface-Comunic, Saúde, Educ. 2010; 14: 593-605.

24. Coutinho SB, Lima MC, Ashworth A, Lira PIC. Babyfriendly hospital initiative and breast feeding practices. $\mathrm{J}$ Pediatr (Rio J). 2005; 81: 471-7.

25. Ortiz PN, Rolim RB, Souza MFL, Soares PL, Vieira TO, Vieira GO, Lyra PPR, Silva LR. Comparação das práticas de amamentação em hospitais IHAC e não credenciados em Salvador, Bahia. Rev Bras Saúde Matern Infant. 2011; 11: 405-13.

26. Oliveira MIC, Silva KS, Gomes-Jr SC, Fonseca VM. Resultado do teste rápido anti-HIV após o parto: uma ameaça à amamentação ao nascimento. Rev Saúde Pública. 2010; 44: 60-9.

27. Meirelles CAB, Oliveira MIC, Mello RR, Varela MAB, Fonseca VM. Justificativas para uso de suplemento em recém-nascidos de baixo risco de um Hospital Amigo da Criança. Cad Saúde Pública. 2008; 24: 2001-12.

28. Abolyan LV. The breast feeding support and promotion in baby-friendly maternity hospitals and not-as-yet babyfriendly hospitals in Russia. Breastfeed Med. 2006; 1: 71-8.

29. Hofvander Y. Breast feeding and the Baby Friendly Hospitals Initiative (BFHI): organization, response and outcome in Sweden and other countries. Acta Paediatr. 2005; 94: 1012-6.

Recebido em 14 de fevereiro de 2012

Versão final apresentada em 4 de julho de 2012

Aprovado em 13 de julho de 2012 